\title{
3D Object Class Geometry Modeling with Spatial Latent Dirichlet Markov Random Fields ${ }^{\star}$
}

\author{
Hanchen Xiong Sandor Szedmak Justus Piater \\ Institute of Computer Science, University of Innsbruck \\ \{hanchen.xiong, sandor.szedmak, justus.piater\}@uibk.ac.at
}

\begin{abstract}
This paper presents a novel part-based geometry model for 3D object classes based on latent Dirichlet allocation (LDA). With all object instances of the same category aligned to a canonical pose, the bounding box is discretized to form a $3 \mathrm{D}$ space dictionary for LDA. To enhance the spatial coherence of each part during model learning, we extend LDA by strategically constructing a Markov random field (MRF) on the part labels, and adding an extra spatial parameter for each part. We refer to the improved model as spatial latent Dirichlet Markov random fields (SLDMRF). The experimental results demonstrate that SLDMRF exhibits superior semantic interpretation and discriminative ability in model classification to LDA and other related models.
\end{abstract}

\section{Introduction}

During the past decades, computer vision has made remarkable progress in visual object understanding, e.g. classification, pose estimation and segmentation, etc. However, most previous study of object modeling is based on 2D images, in which appearance is the main and only information source for various tasks, so most attention is focused on increasing the robustness of algorithms to lighting changes, intra-class appearance variation and viewpoint variation [4]. Meanwhile, 3D geometry properties of objects have been rarely exploited and used to increase the expressiveness of object models. Recently, pioneering work $[7,13]$ has attempted to add 3D geometric information to object models, demonstrating that the accuracy and robustness of such algorithms can be enhanced with extra 3D geometry clues. However, there still exists an obvious gap between $2 \mathrm{D}$ appearance modeling and $3 \mathrm{D}$ geometry modeling with respect to their interpretation and representation abilities, and it has been advocated $[7,13]$ that robust 3D geometry modeling is highly desirable. Motived by this gap and desire, this paper puts forward a novel 3D object class geometry model in the light of state-of-the-art techniques developed in machine learning and computer graphics. Part-based models have displayed merits in 2D appearance modeling [4] for handling partial occlusion, our 3D geometry model is likewise part-based and inherits these strengths. The training data of our algorithm are collections of 3D

\footnotetext{
* The research has received funding from the European Community's Seventh Framework Programme (FP7) under grant agreement no. 270273, Xperience.
} 

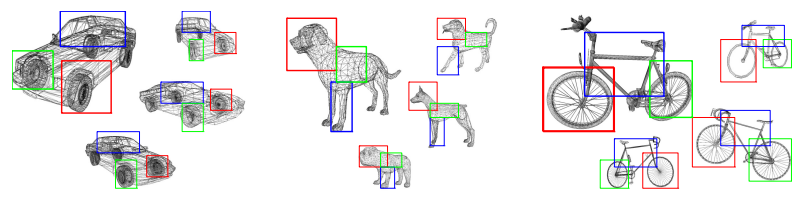

Fig. 1. Different object instances of the same class should share similar 3D structure of composing parts, although their parts can slightly vary from one instance to another.

models of different instances which belong to the same category (Figure 1). The basic underlying principle of our modeling is the concept that different object instances of the same class should share similar 3D structure of composing parts, although their parts can slightly vary from one instance to another. For example, all bicycles are composed of a frame and two wheels, and the geometric relation between these three parts are similar across different instances (Figure 1). In this paper, 3D objects are represented by point cloud data (PCD), which is a general and popular representation of 3D shapes and can easily be converted from other data formats (e.g. meshes). First, for each class, different PCDs of object instances are aligned using point cloud registration methods. Secondly, the main learning step is inspired by latent Dirichlet allocation (LDA) [1] and computer graphics [5]. LDA is a state-of-the-art machine learning tool for discovering latent topics within document collections. Here we apply LDA by considering each object point cloud as a document, and each part as a topic. With the bounding box volume discretized into a 3D grid dictionary, the part can be mined out as a multinomial distribution over the discrete 3D space, and each object is a multinomial distribution over parts. However, standard LDA ignores the spatial coherence, which is of great importance in our task but not generally taken into account in natural language applications. Based on discoveries in computer graphics [5] and other work on LDA [8,11], we develop a spatial latent Dirichlet Markov random field (SLDMRF) model with extra undirected links between topic labels and spatial parameters. The proposed SLDMRF can co-segment all point clouds simultaneously under a prior of coherence of correspondence, spatial continuity and spatial smoothness. According to our empirical results (section 3), compared to standard LDA and other related models, SLDMRF can achieve much more consistent and semantically meaningful segmentations of 3D point clouds, and the learned class geometry models display better discriminative ability in classification.

\subsection{Related work}

The starting point of 3D geometry modeling in visual object understanding is the difficulty in dealing with appearance variation due to different viewpoints. There have been several attempts to embed 3D geometric information into object models $[2,3,7,13]$, and all of them have reported improvement in accuracy and robustness, although different 3D geometry information are exploited and modelled in their work. In [2] 3D object shapes are probabilistically modelled as continuous 
distributions in $\mathbb{R}^{3}$ with a kernel density estimator (KDE). However, that work explicitly addresses neither category-level tasks nor semantic segmentation. Objects are considered as Gausssaian mixtures and expectation-maximization (EM) is applied to estimate corresponding Gaussian parameters and weights. One observation of Gaussian-mixture-based segmentation is that discovered parts rarely display good semantic interpretability since usually the part geometry is too complex to be modelled as a Gaussian (section 3.1). Other work attempts to improve the expressiveness of object geometry models in different ways. For example, Detry et al. [3] represent objects as hierarchically-organized spatial distributions of distinct feature types, but did not seek to produce semantically-meaningful segmentations. Other models $[7,13]$ extract $3 \mathrm{D}$ geometry information at the class level. However, in [7] the segmentation is again based on Gaussian mixtures and EM, and most [13] do not model object classes in a part-based manner to avoid segmentation. Meanwhile, another thread of segmentation-based visual modeling is the application of Latent Dirichlet allocation (LDA) in computer vision $[10,11,8]$. LDA was originally developed to discover hidden topics in text corpora by clustering words into different topics [1]. Standard LDA, however, ignores spatial coherence, which is problematic in vision applications. Therefore, spatial LDA (SLDA) [11] and Latent Dirichlet Markov random fields (LDMRF) [8] were put forward to produce better, spatially-coherent segmentations. In addition, with higher emphasis of the smoothness of parts and consistent correspondences, 3D segmentation in computer graphics [5] constructs graphs with neighboring intra-links and correspondence inter-links among objects, and mincut is used on graphs for segmentation.

The main contribution of this paper is an extension of LDA for 3D object class geometry modeling, which we refer to as Spatial Latent Dirichlet Markov Random Fields(SLDMRF). The proposed model is built with inspiration from recent advances in different fields $[1,11,8,5]$, and it yields superior interpretability and representational capability in modeling $3 \mathrm{D}$ object class geometry.

\section{3D Object Class Geometry Modeling}

With the point cloud representations of 3D object shapes, the alignment of different instances of the same class is achieved with point cloud registration algorithms. While any suitable registration procedure can be used, we adopted a novel method [12] since it is very efficient and robust to non-rigid transformation, which suits the case of intra-category shape variation. An example of aligning dogs is displayed in Figure 2.

\subsection{Latent Dirichlet Allocation}

LDA [1] is a generative model that utilizes the information of co-occurring words to find out hidden topics shared by documents. In LDA, each document is considered as a finite mixture of topics; each topic is a finite mixture of words. The graphical model of LDA is shown in Figure 3(a). The generative process of LDA 


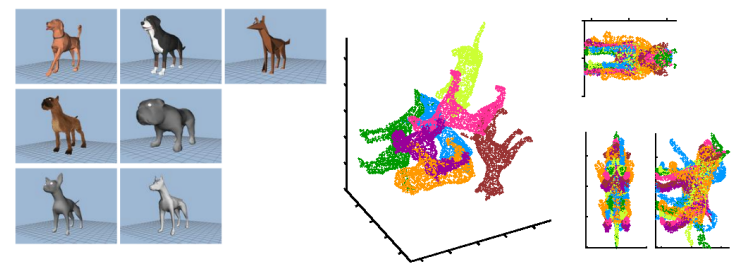

Fig. 2. Alignment of different dog instances by point cloud registration [12]. Left: original 3D shapes of different dog instances; middle: point clouds generated from the shapes on the left; right: three views (top, profile, front) of the point clouds (middle) after alignment.

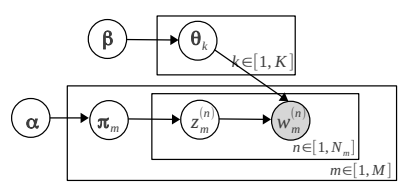

(a)

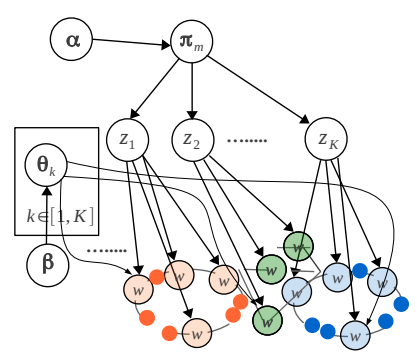

(b)

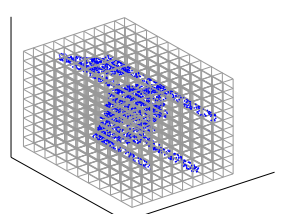

(c)

Fig. 3. (a) Graphical model of LDA; (b) Application of LDA to model 3D object categories; (c) Construction of 3D dictionary by discretizing the 3D space of the bounding box.

is as follows: (1) for each topic $k \in[1, K]$, a multinomial parameter $\boldsymbol{\theta}_{k}$ over words is sampled from Dirichlet prior $\boldsymbol{\beta} ;(2)$ for each document $m \in[1, M]$, a multinomial parameter $\boldsymbol{\pi}_{m}$ over $K$ topics is sampled from Dirichlet prior $\boldsymbol{\alpha}$; (3) for each word $w_{m}^{(n)}, n \in\left[1, N_{m}\right]$ in document $m$, a topic label $z_{m}^{(n)}$ is first sampled from multinomial distribution $z_{m}^{(n)} \sim \operatorname{Multinomial}\left(\boldsymbol{\pi}_{m}\right)$, then the word $w_{m}^{(n)}$ is sampled from the multinomial distribution parametrized with $\boldsymbol{\theta}_{z_{m}^{(n)}}$, $w_{m}^{(n)} \sim \operatorname{Multinomial}\left(\boldsymbol{\theta}_{z_{m}^{(n)}}\right)$. Hyperparameters $\boldsymbol{\alpha}$ and $\boldsymbol{\beta}$ define the Dirichlet priors governing the parameters of multinomial distributions. Usually $\boldsymbol{\alpha}$ and $\boldsymbol{\beta}$ are set in a symmetric manner and using low values [6]. In [10], LDA is applied on a collection of images. Each image is considered as a document, objects correspond to topics, and visual words are generated using vector quantization on extracted features. In our case, however, LDA is utilized for 3D object class geometry modeling with objects of the same category as documents, and parts shared by different instances correspond to topics (Figure 3(b)).

3D Dictionary. In our task, LDA is expected to work effectively under the assumption that different objects of the same category should share very similar structure. Therefore, when LDA is applied on each collection of categorical object 
point clouds, the co-occurring patterns are the 3D space occupied by 3D points. In each collection, all instances can be aligned to a canonical pose, based on which the $3 \mathrm{D}$ space of the bounding box is discretized into a grid, where each block represents a 3D word. Therefore, when transferring point clouds to corresponding documents, word $w_{k}$ will replace 3D point $x_{i}$ if $x_{i}$ lies within block $w_{k}$. In this way, the discovered part actually is a distribution over 3D space, and a category is a mixture of these distributions. The concept of dictionary discretization is illustrated in Figure 3(c).

For label inference and parameters learning in LDA, a collapsed Gibbs sampling [6] can be formulated as

$$
q_{\mathrm{LDA}}\left(z_{m}^{(n)}=k\right) \propto \frac{\mathbf{N}_{-m n, w_{m}^{(n)}}^{(k)}+\beta_{w_{m}^{(n)}}}{\sum_{w}^{W}\left(\mathbf{N}_{-m n, w}^{(k)}+\beta_{w}\right)} \cdot\left(\mathbf{N}_{-m n, k}^{(m)}+\alpha_{k}\right)
$$

where $\mathbf{N}_{-m n, w}^{(k)}$ is the number of words in the corpus with value $w$ assigned to topic $k$ excluding the $n$th word in document $m$, and $\mathbf{N}_{-m n, k}^{(m)}$ is the number of words in document $m$ assigned to topic $k$ excluding the $n$th word in document $m$. From (1), it can be seen that LDA prefers to cluster together those words that often co-occur in the same document. Therefore, simply applying LDA on the 3D dictionary, unfortunately, is not expected to work because it misses a lot of spatial and correspondence information, which is not meaningful in the text processing case: (1) Spatial coherence is an important issue when LDA is applied in vision applications $[11,8]$. For example, in all point clouds of dogs, 3D words located in the hip and in the head will always co-occur. So by using (1), the hip and head of dogs can be clustered into a part, which is a spatially (of course also semantically) unreasonable segmentation. (2) Correspondence coherence is likewise important. LDA can find synonyms by finding their co-occurring patterns in documents. However, the "synonyms" in the 3D dictionary are identified by spatial correspondence. For example, in Figure 2, the legs of different dogs can rarely match exactly due to different species or standing poses. However, since all legs are close and correspond to each other, they should be clustered into the same part.

\subsection{Spatial latent Dirichlet Markov random field}

To enhance the spatial coherence, in Spatial LDA (SLDA) [11] 2D images are decomposed into small overlapping regions, which are used as documents to ensure that the pixels belonging to one part should be close to each other. Latent Dirichlet Markov random fields (LDMRF) [8], on the other hand, construct Markov random fields on the part label variables to enhance the local spatial coherence. However, both of them ignore the correspondences across the segmentations of different instances. Inspired by these improved versions of LDA and consistent co-segmentation in computer graphics [5], we put forward a novel spatial latent Dirichlet Markov random field (SLDMRF) that inherits virtues from both SLDA and LDMRF. However, rather than being a simple combination of SLDA and LDMRF, the proposed SLDMRF goes beyond them in several ways. 


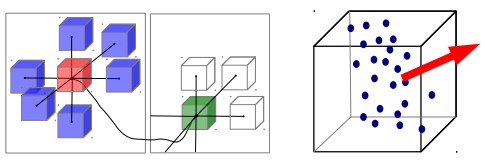

(a) Left: for each word (red block), there are two types of connections in SLDMRF: neighboring spatial connections (blue blocks) and correspondence connections (green block); right: the normal vector of each word in the document is estimated by using the points lying within the word.

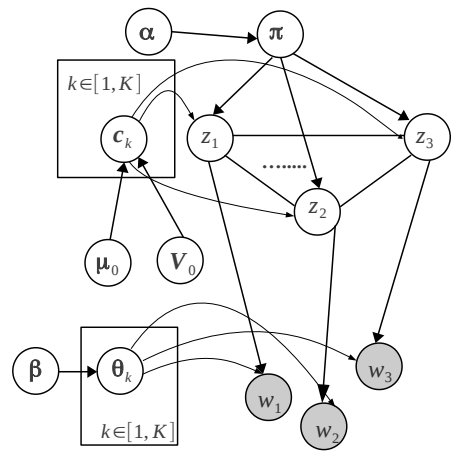

(b) Graphical model of SLDMRF: compared to the standard LDA (Figure 3(a)), there are extra directed links between topic labels and spatial Gaussian parameters $\mathbf{c}_{k}$.

Fig. 4. SLDMRF modeling

First, instead of going through all small overlapping sub-volumes as SLDA does, we explicitly model the positions of all parts by parameters $\mathbf{c}_{k}$ such that 3D words that share the same label $k$ are likely to be close to $\mathbf{c}_{k}$. Second, similarly to LDMRF, SLDMRF constructs a Markov random field on the neighboring label variables. However, different from LDMRF, we assign different potential functions based on the prior that the segmentation boundaries should be located at the point where abrupt curvature changes take place. The potential function is defined as

$$
g\left(z_{i}, z_{j}\right)=\delta\left(z_{i}, z_{j}\right) \exp \left(\left|\left\langle o_{i}, o_{j}\right\rangle\right|\right)
$$

where $\delta\left(z_{i}, z_{j}\right)$ equals 1 when $z_{i}$ and $z_{j}$ are neighbors, and 0 otherwise (Figure $4(\mathrm{a}))$, and $o_{i}, o_{j}$ are the normal vectors estimated by using the points lying within word $i$ and $j$ respectively (Figure 4(a)). Last but not least, SLDMRF enhance the correspondences of segmentation across different instances. Inspired by the co-segmentation used in [5], we construct inter-connections between corresponding parts across different objects, and correspondences are matched by finding the nearest neighbors in other objects after alignment. In this way the segmentation can be more consistent within a category. The potential function $g\left(z_{m}^{(i)}, z_{n}^{(j)}\right)$ for correspondence connections is set in the same way as spatial connections $(2) ; \delta\left(z_{m}^{(i)}, z_{n}^{(j)}\right)$ is 1 if $z_{m}^{(i)}$ and $z_{n}^{(j)}$ are nearest neighbours of each other, and 0 otherwise. Because the part weights are already taken into account by LDA (parameter $\boldsymbol{\pi}$ ), the labels within the Markov random fields are modeled as:

$$
p(\mathbf{Z}) \propto \exp \left(\sum_{(i, j)} g\left(z_{i}, z_{j}\right)\right)
$$

The graphical model of SLDMRF is presented in Figure 4(b). Hyperparameters $\boldsymbol{\mu}_{0}$ and $\mathbf{V}_{0}$ (similar to $\boldsymbol{\alpha}, \boldsymbol{\beta}$ ) specify the Gaussian prior of part po- 
sitions $\mathbf{c}_{k} \sim \mathcal{N}\left(\cdot ; \boldsymbol{\mu}_{0}, \mathbf{V}_{0}\right)$. Given the part position $\mathbf{c}_{k}$, the label is sampled as $z_{i} \sim \mathcal{N}\left(\hat{w}_{i} ; \mathbf{c}_{k}, \boldsymbol{\Lambda}\right)$, where $\hat{w}_{i}$ denotes the $3 \mathrm{D}$ coordinates of word $w_{i}$. Since we do not expect the label distribution to be truly Gaussian, $\boldsymbol{\Lambda}$ is set relatively large. The joint probability of $3 \mathrm{D}$ words in $\operatorname{SLDMRF} p\left(\left\{w_{m}^{(n)}\right\}_{m=1, n=1}^{M, N_{m}}, \mid \boldsymbol{\alpha}, \boldsymbol{\beta}\right)$ is:

$$
\begin{gathered}
\frac{1}{\mathbf{Q}} \prod_{m=1}^{M} \prod_{n=1}^{N_{m}}\left(\int _ { \boldsymbol { \pi } _ { m } } \int _ { \boldsymbol { \theta } _ { z _ { m } ^ { ( n ) } } } \int _ { \mathbf { c } _ { z _ { m } ^ { ( n ) } } } p ( \boldsymbol { \pi } _ { m } | \boldsymbol { \alpha } ) \sum _ { z _ { m } ^ { ( n ) } = 1 } ^ { K } \left(p\left(z_{m}^{(n)} \mid \boldsymbol{\pi}_{m}\right) p\left(w_{m}^{(n)} \mid \boldsymbol{\theta}_{z_{m}^{(n)}}\right)\right.\right. \\
\left.\left.\mathcal{N}\left(w_{m}^{(n)} ; \mathbf{c}_{z_{m}^{(n)}}, \boldsymbol{\Lambda}\right) \mathcal{N}\left(\mathbf{c}_{z_{m}^{(n)}} ; \boldsymbol{\mu}_{0}, \mathbf{V}_{0}\right)\right) \times \prod_{x, y \in \tilde{z}_{m}^{(n)}} \sqrt{\exp \left(g\left(z_{m}^{(n)}, z_{x}^{(y)}\right)\right)}\right)
\end{gathered}
$$

where $x, y \in \tilde{z}_{m}^{(n)}$ denotes the set of all word labels (the $y$ th word in the $x$ th document) connected with $z_{m}^{(n)}$, i.e. $\delta\left(z_{m}^{(n)}, z_{x}^{(y)}\right)=1$, and $\mathbf{Q}$ is the normalization term induced by Markov random fields.

\subsection{Inference and learning}

Similar to the inference and learning in LDA, based on (4), we can develop a collapsed Gibbs sampler by integrating out $\boldsymbol{\pi}_{m}, \boldsymbol{\theta}_{z_{m}^{(n)}}, \mathbf{c}_{z_{m}^{(n)}}$ in SLMRF. The sampler can be more easily interpreted as a "combined" sampler by using clues from LDA, MRF and Guassian mixtures:

$$
q^{*}\left(z_{m}^{(n)}=k\right) \propto q_{\mathrm{LDA}}\left(z_{m}^{(n)}=k\right) \cdot q_{M}\left(z_{m}^{(n)}=k\right) \cdot q_{c}\left(z_{m}^{(n)}=k\right)
$$

where $q_{\mathrm{LDA}}\left(z_{m}^{(n)}=k\right)$ is the collapsed Gibbs sampler of LDA (1),

$$
q_{M}\left(z_{m}^{(n)}=k\right) \propto \frac{\exp \left(\sum_{\left(z_{j}, z_{m}^{(n)}\right)} g\left(z_{j}, z_{m}^{(n)}=k\right)\right)}{\sum_{h} \exp \left(\sum_{\left(z_{j}, z_{m}^{(n)}\right)} g\left(z_{j}, z_{m}^{(n)}=h\right)\right)}
$$

is the Gibbs sampler based on the Markov random field, and

$$
q_{c}\left(z_{m}^{(n)}=k\right) \propto \frac{\mathcal{N}\left(\hat{w}_{m}^{(n)} ; \boldsymbol{\mu}_{l}^{(k)}, \boldsymbol{\Lambda}_{l}^{(k)}\right)}{\sum_{h} \mathcal{N}\left(\hat{w}_{m}^{(n)} ; \boldsymbol{\mu}_{l}^{(h)}, \boldsymbol{\Lambda}_{l}^{(h)}\right)}
$$

is a collapsed Gibbs sampler of Gaussian mixtures, with

$$
\boldsymbol{\Lambda}_{l}^{(k)^{-1}}=\boldsymbol{\Lambda}_{0}^{-1}+l \boldsymbol{\Lambda}^{-1} \quad \boldsymbol{\mu}_{l}^{(k)}=\boldsymbol{\Lambda}_{l}^{(k)^{2}}\left(\frac{\boldsymbol{\mu}_{0}}{\boldsymbol{\Lambda}_{0}^{2}}+\frac{\overline{l \hat{w}^{(k)}}}{\boldsymbol{\Lambda}^{2}}\right)
$$

where $l$ is the number of words which are labeled with $k$, and $\overline{\hat{w}^{(k)}}$ is the mean of $3 \mathrm{D}$ coordinates of words which are assigned to part $k$ until the current iteration. Similar to [6], parameters $\left\{\boldsymbol{\pi}_{m}\right\}_{m=1}^{M},\left\{\boldsymbol{\theta}_{k}\right\}_{k=1}^{K}$ can be estimated after the convergence of Gibbs sampling:

$$
\boldsymbol{\pi}_{m}^{(k)}=\frac{\mathbf{N}_{k}^{(m)}+\alpha_{k}}{\sum_{k=1}^{K}\left(\mathbf{N}_{k}^{(m)}+\alpha_{k}\right)} \quad \boldsymbol{\theta}_{k}^{(w)}=\frac{\mathbf{N}_{w}^{(k)}+\beta_{w}}{\sum_{w}^{W}\left(\mathbf{N}_{w}^{k}+\beta_{w}\right)}
$$

Since hyperparameter $\boldsymbol{\alpha}$, in our case, is categorical part weight, we estimate it by simply compute the average of $\pi_{m}: \boldsymbol{\alpha}=\frac{1}{M} \sum_{m=1}^{M} \boldsymbol{\pi}_{m}$. In addition, parameters $\left\{\mathbf{c}_{k}\right\}_{k=1}^{K}$ are read out as $\left\{\boldsymbol{\mu}_{l}^{k}\right\}_{k=1}^{K}(8)$. 


\section{Experiments}

To evaluate the proposed model, 5 object classes (cars, bikes, dogs, motorcycles, airplanes) from the Princeton shape benchmark (PSB) [9] database are used. Since 3D shapes in the PSB are represented as triangulated meshes, we convert them to point clouds by uniformly sampling points within triangles.

\subsection{D Object class geometry modeling}

For comparison, LDA [1], LDMRF [8] and Gaussian Mixtures (GM) models are tested on the same data (aligned point clouds of categorical instances). Since LDMRF requires manual interference (semi-supervision), to avoid human bias during comparison, here we construct the same Markov random fields for both LDMRF and SLDMRF so that LDMRF can also work in an unsupervised manner. All four models are implemented with Gibbs sampling for label inference and learning. To ensure fairness, the same part number and iteration number (200) is applied. A test example of motorcycle modeling is presented in Figure 5. LDA does not find consistent and meaningful parts because of the intra-class variation (each object is labeled as a part since LDA only focuses on co-occurring patterns). LDMRF, on the other hand, discovers some locally continuous and consistent segments on different objects. However, the global spatial coherence of parts is poor; parts are shattered. GM establishes more globally obvious segmentation pattern by finding more consistent and meaningful parts. Nevertheless, GM ignores local spatial coherence, so parts are not well segmented; they tend to be of blob shape and to overlap each other. By contrast, SLDMRF produces best convincing segmentations in terms of consistence, local and global spatial coherence and semantic meaning. The SLDMRF modeling results of other four object classes are illustrated in Figure 6.

\subsection{Geometry model classification}

To illustrate the parts learned by SLDMRF is more accurate, and thus more discriminative, we conduct quantitative comparisons on classification task. Since LDA and LDMRF are far from being qualified for practical part-based modeling, here we are only concerned with the comparison between GM and SLDMRF. 3D shapes of 5 object classes are divided into training (70\%) and test sets (30\%). The model learning is conducted in the same way as in section 3.1. Although Markov random fields and spatial parameters greatly assists in segmentation and model learning of SLDMRF, they are not used in the final category models. A learned bicycle model is shown in Figure 6(e). It can be seen that the part position information and neighboring correlation are already encoded in the categorical part parameter $\boldsymbol{\theta}$. Therefore, for the sake of simplicity and computation feasibility, we only use learned LDA as our 3D object category models for classification. To test an object $M^{*}$, it is first aligned to the canonical poses of different class models. In SLDMRF case, the likelihood that $M^{*}$ belongs to a 


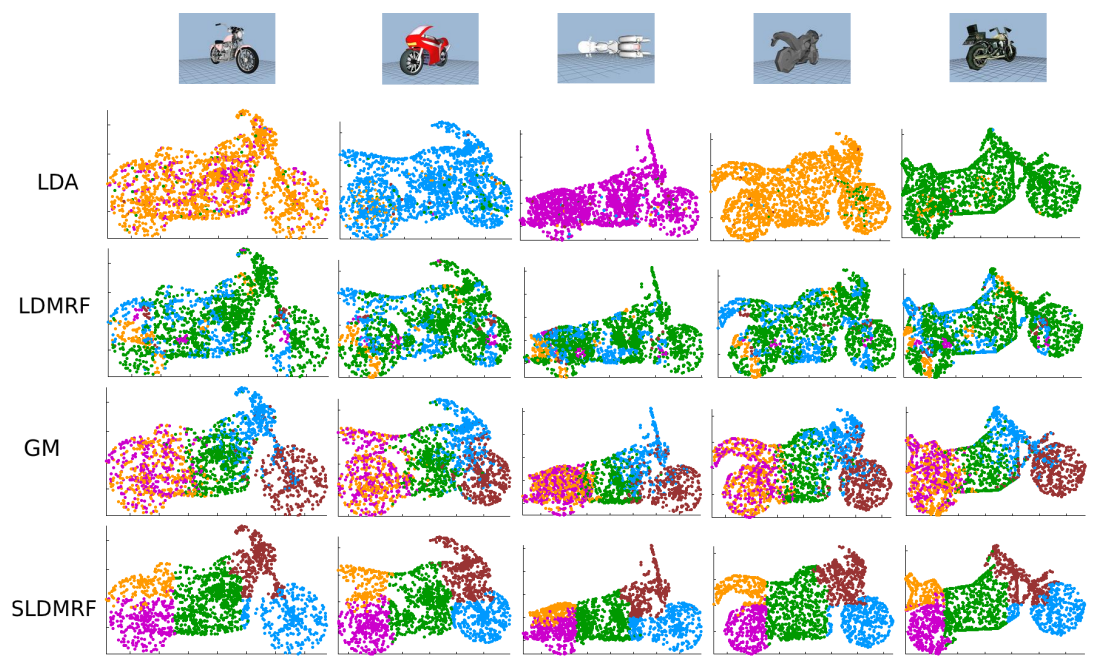

Fig. 5. Comparison of segmentation by using LDA, LDMRF, GM and SLDMRF, SLDMRF qualitatively yields more reasonable segmentations than the others.

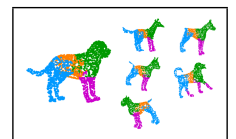

(a)

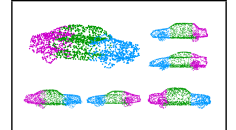

(b)

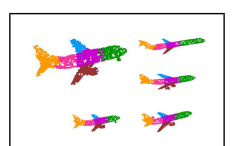

(c)

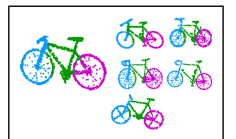

(d)

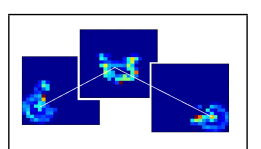

(e)

Fig. 6. SLDMRF modeling of dogs (a), cars (b), airplanes (c) and bikes (d); (e): the learned part parameters $\boldsymbol{\theta}_{k}$ of bikes.

class $x \in\{$ cars, bikes, dogs, motorcycles, airplanes $\}$ is computed as:

$$
p\left(M^{*} \mid \mathcal{M}_{x}\right)=\prod_{i=1}^{\left|M^{*}\right|}\left\{\sum_{k} \int_{\boldsymbol{\pi}} p\left(w_{i} \mid \boldsymbol{\theta}_{k}\right) p(k \mid \boldsymbol{\pi}) p(\boldsymbol{\pi} \mid \boldsymbol{\alpha})\right\}
$$

where $\left|M^{*}\right|$ is the number of points in object $M^{*}$. By contrast, in the GM case:

$$
p\left(M^{*} \mid \mathcal{M}_{x}\right)=\prod_{i=1}^{\left|M^{*}\right|}\left\{\sum_{k} \mathcal{N}\left(w_{i} ; \boldsymbol{\theta}_{k}\right) \pi_{k}\right\}
$$

Since no other prior knowledge is given, the classification can be done in a maximum-likelihood fashion. A global model learned with one single multinomial distribution on 3D dictionary is also provided as baseline for comparison. The classification performances of GM, SLDMRF and global model are evaluated using confusion matrices. The comparison in in Figure 7 demonstrates that SLDMRF is superior to GM with respect to discriminative ability in classification. 


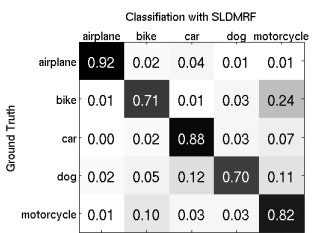

(a)

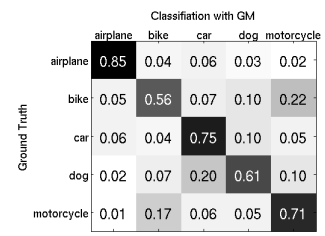

(b)

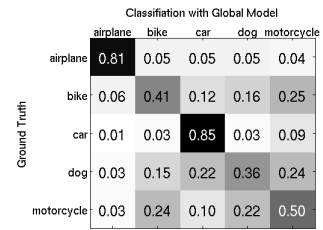

(c)

Fig. 7. The classification confusion matrcices of 5 object classes with SLDMRF (a), GM (b) and global model (c).

\section{Conclusion and Discussion}

We improved LDA model for geometry modeling with better semantic interpretation and promising discriminative capabilities. Meanwhile, learning and application of the model require good initial alignment, which is difficult for noisy and partial occluded 3D point cloud in practice. So a promising future work is to cooperate 3D geometry model with 2D image models to describe both structure and appearance, which thus enhance model's expressiveness and practical value.

\section{References}

1. Blei, D., Ng, A., Jordan, M.: Latent Dirichlet Allocation. Journal of Machine Learning Research 3, 993-1022 (2003)

2. Detry, R., Piater, J.: Continuous Surface-Point Distributions for 3D Object Pose Estimation and Recognition. In: ACCV (2010)

3. Detry, R., Pugeault, N., Piater, J.: A Probabilistic Framework for 3D Visual Object Representation. PAMI 31(10), 1790-1803 (10 2009)

4. Felzenszwalb, P.F., Girshick, R.B., McAllester, D., Ramanan, D.: Object Detection with Discriminatively Trained Part-Based Models. PAMI 32(9), 1627-1645 (2010)

5. Golovinskiy, A., Funkhouser, T.A.: Consistent segmentation of 3D models. Computers and Graphics 33, 262-269 (2009)

6. Griffiths, T.L., Steyvers, M.: Finding scientific topics. Proceedings of the National Academy of Sciences 101(Suppl. 1), 5228-5235 (April 2004)

7. Jörg Liebelt and Cordelia Schmid: Multi-View Object Class Detection with a 3D Geometric Model. In: CVPR (2010)

8. Mackey, L.: Latent Dirichlet Markov Random Fields for Semi-supervised Image Segmentation and Object Recognition (2007)

9. Shilane, P., Min, P., Kazhdan, M.M., Funkhouser, T.A.: The Princeton Shape Benchmark. In: SMI. pp. 167-178. IEEE Computer Society (2004)

10. Sivic, J., Russell, B.C., Efros, A.A., Zisserman, A., Freeman, W.T.: Discovering object categories in image collections. In: ICCV (2005)

11. Wang, X., Grimson, E.: Spatial Latent Dirichlet Allocation. In: NIPS (2007)

12. Xiong, H., Szedmak, S., Piater, J.: Efficient,General Point Cloud Registration with Kernel Feature Maps. In: Canadian Conf. on Computer and Robot Vision (2013)

13. Yan, P., Khan, S.M., Shah, M.: 3D model based object class detection in an arbitrary view. In: ICCV (2007) 